\title{
Silent ST Segment Elevation Myocardial Infarction with Multi-segmental Renal Infarction: An Unusual Presentation
}

\author{
Hung-Yu Chang and Yung-Nien Yang
}

\begin{abstract}
A 36-year-old diabetic man came to our institution presenting with constant left flank pain. Left renal embolic infarction was found by abdominal computed tomography. Silent ST segment elevation myocardial infarction was noted on 12-lead electrocardiogram. Emergent coronary angiography revealed large thrombus burdens with complete occlusion at the left anterior descending artery ostium, which may be the embolic origin. Silent ST segment elevation myocardial infarction with acute flank pain and multiple segmental renal infarction is an unusual presentation. High vigilance may prevent delay of the "golden hour" to treat acute myocardial infarction.
\end{abstract}

Key words: renal infarction, myocardial infarction, percutaneous coronary intervention

(Intern Med 50: 723-725, 2011)

(DOI: 10.2169/internalmedicine.50.4517)

\section{Introduction}

Renal infarction is an easily-missed disease. It often occurs by thromboembolism in patients with electrical or structural heart disease, but it can also happen with homocysteinemia, malignancy, coagulopathy, trauma, invasive iatrogenic procedure or cocaine abuse. The potential sources of peripheral emboli include intra-cardiac thrombus, infective endocarditis, intra-coronary thrombus and aortic atherosclerotic plaque. We report a case with silent ST segment elevation myocardial infarction with the initial presentation of left flank pain and multiple segmental left renal embolic infarction.

\section{Case Report}

A 36-year-old man presented at our institution with sudden onset of constant left flank pain for 10 hours, associating with left lower abdominal pain and dysuria. He has smoked cigarettes for more than 20 years and has a history of type 1 diabetes with regular medical treatment, but he denied a history of urolithiasis. He was afebrile, his heart rate was 102 beats per minute, blood pressure was 140/78 $\mathrm{mmHg}$ and respiratory rate was 14 breaths per minute. On physical examination, the first and second heart sound was normal but the fourth heart sound was presented; the heart rhythm was regular without audible murmur. The abdomen was soft on palpation with mild tenderness in the left lower quadrant but without significant flank knocking pain. Initial studies of complete blood count, serum creatinine, electrolytes, and kidney-ureter-bladder radiography were unremarkable. Serum lactate dehydrogenase level was 278 IU/L (normal 98-192 IU/L). Dipstick urinalysis showed glucosuria, mild proteinuria and slight microscopic hematuria. The patient was still symptomatic after medication. Contrastenhanced abdominal computed tomography revealed peripheral multiple patch and wedge-shaped lesions in the left renal cortex (Fig. 1). Regarding the search for the origin of renal embolic infarction, 12-lead electrocardiography showed sinus tachycardia and left anterior fascicular block associated with ST segment elevation of 2-4 $\mathrm{mm}$ in precordial leads $V_{1}$ to $V_{4}$ (Fig. 2). Transthoracic echocardiography demonstrated left ventricular apical and anteroseptal akinesis without thrombus formation. Left ventricular ejection fraction was 30\%. Emergent coronary angiography showed large thrombus burdens with complete occlusion at the left anterior descending artery ostium (Fig. 3), and 50-60\% stenosis at the left circumflex artery and right coronary artery. Thrombolysis in myocardial infarction (TIMI) III flow was 


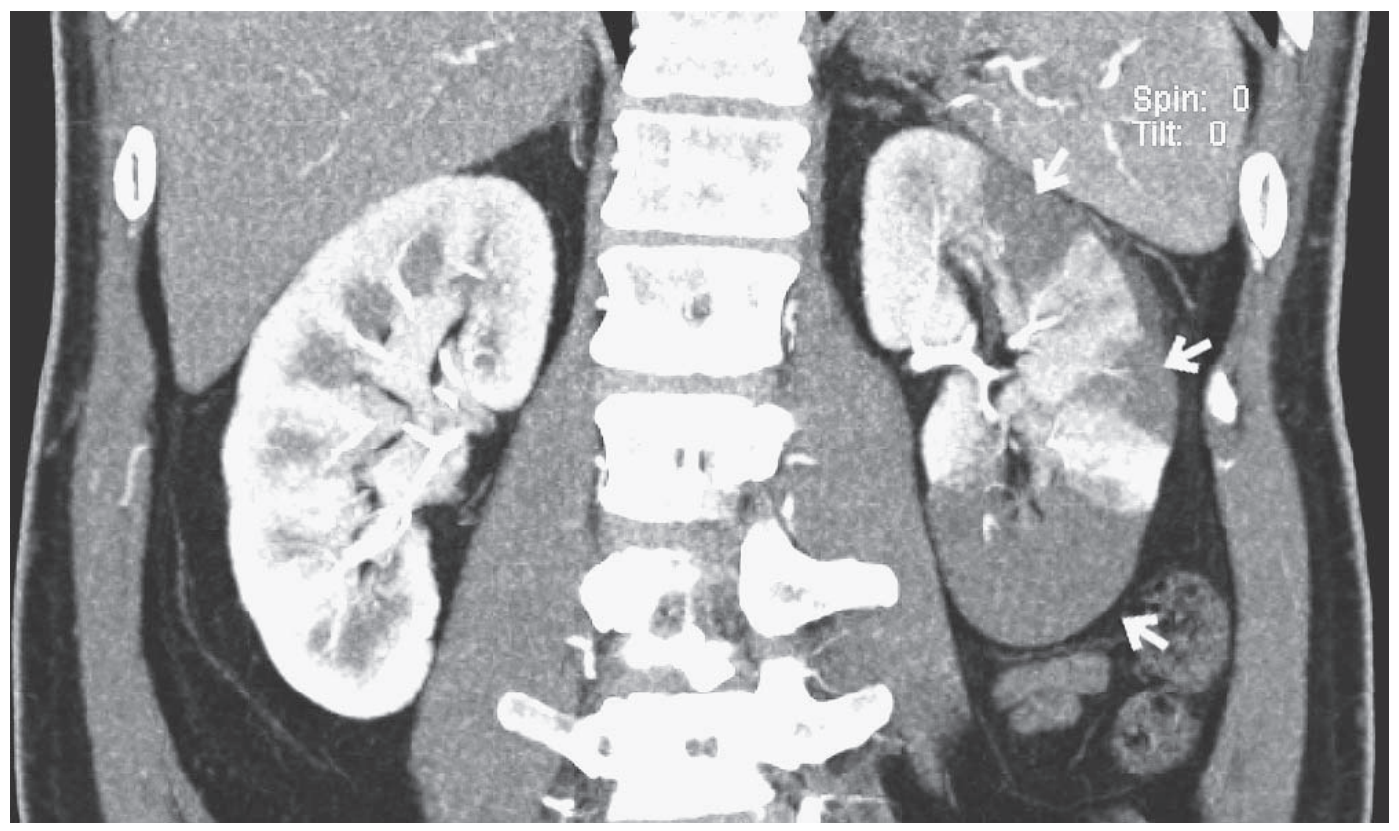

Figure 1. Contrast-enhanced abdominal computed tomography, reconstructed coronal view shows multiple peripheral wedge-like hypodensities involving the superior, middle and inferior portions of the left kidney (arrows), consistent with left embolic renal infarction.

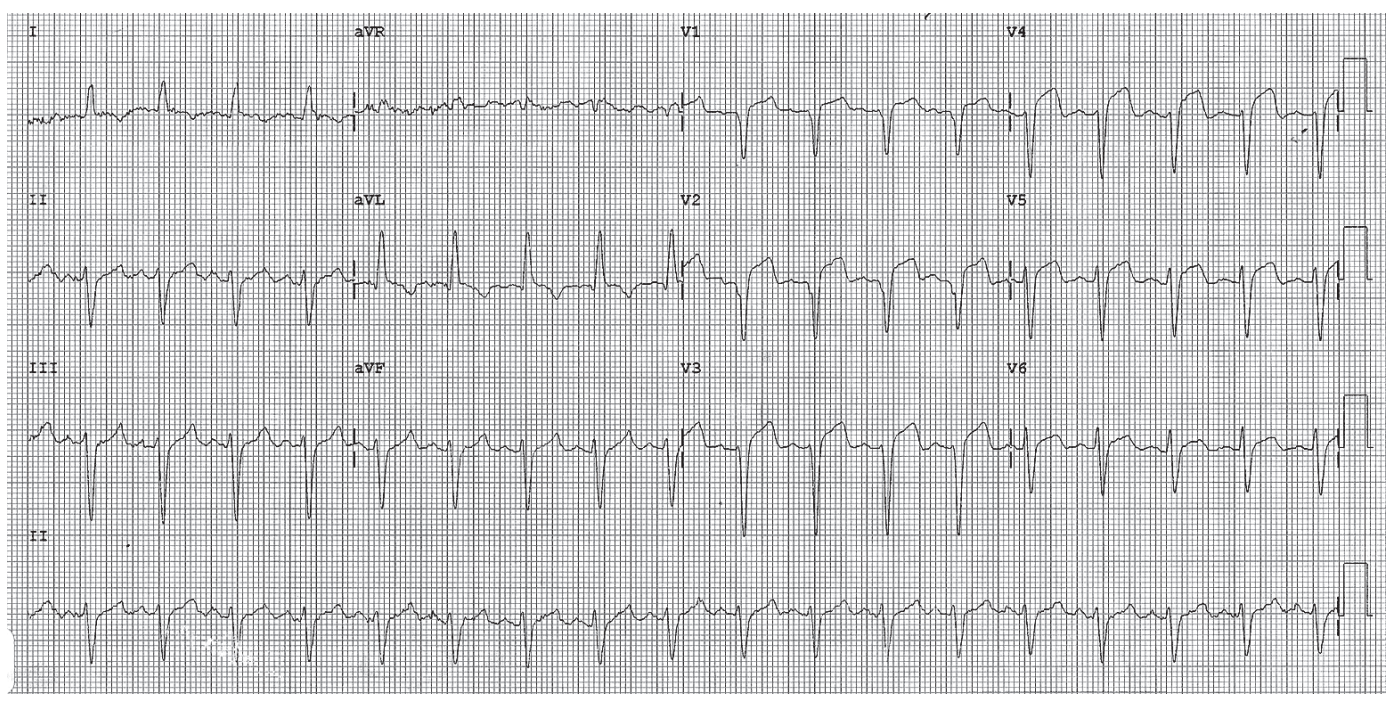

Figure 2. Resting 12-lead electrocardiogram reveals sinus tachycardia, left anterior hemiblock and ST segment elevation of 2-4 $\mathrm{mm}$ in precordial leads $V_{1}$ to $V_{4}$.

achieved after manual thrombus aspiration and balloon angioplasty. Peak post-procedural troponin-I and creatine kinease were $20.45 \mathrm{ng} / \mathrm{mL}$ and $833 \mathrm{IU} / \mathrm{L}$, respectively. The patient was administered aspirin and unfractionated heparin, followed by warfarin. Serial electrocardiography showed resolution of ST segment elevation. There was no evidence of atrial fibrillation during hospitalization and at follow-up. The levels of protein $\mathrm{C}$, protein $\mathrm{S}$ and anti-thrombin III were within the normal range at follow-up.

\section{Discussion}

Renal infarction is an easily-missed disease. The estimated incidence of renal infarction has been reported to be
$0.004-0.007 \%$ in the emergency department $(1,2)$. Previous studies suggest that renal infarction often occurs in patients with electrical or structural heart disease, such as atrial fibrillation, intra-cardiac thrombus, valvular heart disease or infective endocarditis. Renal infarction in patients without heart disease, so-called idiopathic renal infarction, may associate with homocysteinemia, malignancy or other coagulopathy $(3,4)$. Additionally, renal infarction can also be caused by trauma, iatrogenic procedure or cocaine abuse. Most patients with renal infarction are in the sixth decade of life. Clinical presentation of persisting abdominal or flank pain, elevated serum level of lactate dehydrogenase, microscopic hematuria or proteinuria and a high probability of thromboembolic event may suggest renal infarction but these are 


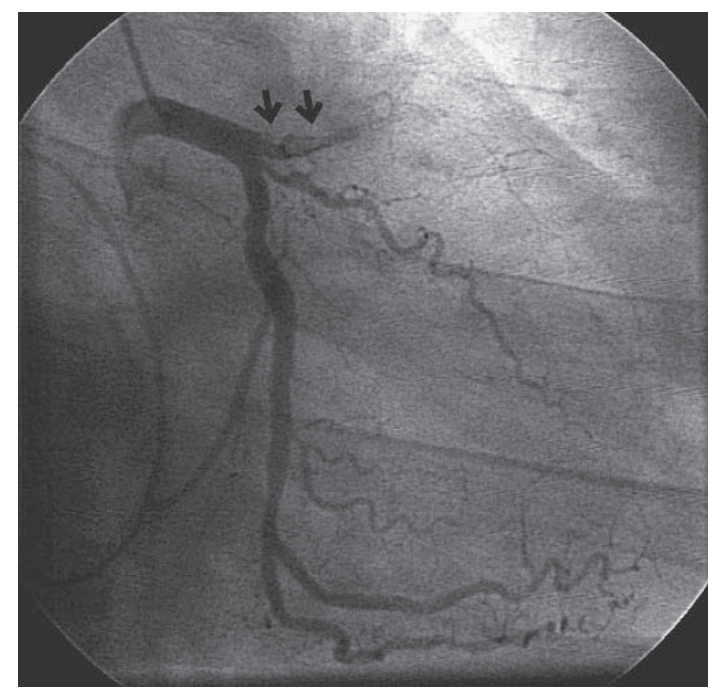

Figure 3. Coronary angiography in right anterior oblique and caudal projections reveal large thrombus burdens with complete occlusion at the left anterior descending artery ostium (arrows).

not specific findings. Contrast-enhanced computed tomography is the first choice in suspected renal infarction based on its convenience and accuracy.

Acute embolic renal infarction could result from left ventricular thrombus, intra-coronary thrombus or disrupted aortic atherosclerotic plaque. Left ventricular mural thrombus, generally visualized between 2 and 11 days after acute myocardial infarction (5), is a well-known risk factor for systemic embolism. However, in the present patient renal in- farction presented relatively early, and there was no detectable mural thrombus by echocardiography, rendering this possibility doubtful. Since large thrombus burdens were seen at the ostium of the left anterior descending artery, the conjecture of the intra-coronary thrombus to cause renal infarction is possible but not probable. In conclusion, acute renal infarction is rare and easily missed. We present this patient with acute flank pain, multiple embolic renal infarction and silent ST segment elevation myocardial infarction, which is certainly an unusual presentation. High vigilance for this rare condition may prevent delay of the "golden hour" to treat acute myocardial infarction.

The authors state that they have no Conflict of Interest (COI).

\section{References}

1. Huang CC, Lo HC, Huang HH, et al. ED presentations of acute renal infarction. Am J Emerg Med 25: 164-169, 2007.

2. Korzets Z, Plotkin E, Bernheim J, Zissin R. The clinical spectrum of acute renal infarction. Isar Med Assoc J 4: 781-784, 2002.

3. Hazanov N, Somin M, Attali M, et al. Acute renal embolism. Forty-four cases of renal infarction in patients with atrial fibrillation. Medicine 83: 292-299, 2004.

4. Bolderman R, Oyen R, Verrijcken A, Knockaert D, Vanderschueren S. Idiopathic renal infarction. Am J Med 119: 356.e9-e12, 2006.

5. Nihoyannopoulos P, Smith GC, Maseri A, Foale RA. The nature history of left ventricular thrombus in myocardial infarction: a rationale in support of masterly inactivity. J Am Coll Cardiol 14: 903-911, 1989.

\footnotetext{
(C) 2011 The Japanese Society of Internal Medicine http://www.naika.or.jp/imindex.html
} 